\title{
Performance analysis of NOMA-based mobile edge computing with imperfect CSI
}

Huan Jiang ${ }^{1,2^{*}}$ (D), Yafei Wang ${ }^{1,2}$, Xinwei Yue ${ }^{1,2}$ and Xuehua Li $i^{1,2}$

\section{*Correspondence:}

jianghuan@mail.bistu.edu.cn

'School of Information and

Communication Engineering,

Beijing Information Science and

Technology University, Beijing,

China

${ }^{2}$ Key Laboratory of Modern

Measurement \& Control

Technology, Ministry of Education, Beijing, China

\section{算 Springer Open}

\begin{abstract}
In this paper, a non-orthogonal multiple access (NOMA)-based mobile edge computing (MEC) system is proposed, where paired users (the $m$ th user and the $n$th user) offload tasks to the MEC server under imperfect channel state information (ipCSI) condition. To evaluate the effect on performance under ipCSI condition in NOMA-MEC system, this study derives new exact and asymptotic expressions of the offloading outage probability for two users under ipCSI and perfect channel state information (pCSI) conditions. On the basis of the theoretical derivation, the diversity orders of the $n$th user under ipCSI and pCSI conditions are zero and $n$, respectively, while those for the distant user are all zeros. In addition, we also investigate the system throughput and energy efficiency of NOMA-MEC in delay-limited transmission. Numerical results show that (1) the offloading outage behaviors of NOMA-MEC are better than those of time division multiple address (TDMA), (2) the offloading outage performance of paired users under ipCSI is worse than that under pCSI, and (3) the NOMA-MEC system achieves higher throughput and energy efficiency than does the TDMA-MEC system.
\end{abstract}

Keywords: Mobile edge computing, Non-orthogonal multiple access, Imperfect channel state information, Offloading outage probability

\section{Introduction}

Future wireless communication will face the challenges of network requirements, such as high-density users, high speed, and ultra-reliable and low latency communications (URLLC). The latency of URLLC is as low as $1 \mathrm{~ms}$, and the reliability is as high as $99.999 \%$, which can ensure mission-critical communications. In the future 6th generation networks, URLLC will provide powerful guarantee for emerging applications that have strict requirements on latency and reliability [1]. Traditional orthogonal multiple access (OMA) divides physical resources into orthogonal resource blocks in time/frequency/code domains, which limits the number of accessing users. To meet the high requirements of communication networks, highly efficient multiple access technologies are required to improve the system capacity and spectrum utilization under limited spectrum resources. Non-orthogonal multiple access (NOMA) ${ }^{1}$ has become a popular research topic in

\footnotetext{
${ }^{1}$ The superposed signals for multiple users can be mapped to single physical resource element or multiple elements. Based on these, NOMA can be divided into single carrier NOMA (SC-NOMA) and multi-carrier NOMA [2]. In this paper, we focus on single carrier NOMA and use NOMA to represent SC-NOMA.
}

(c) The Author(s). 2020 Open Access This article is licensed under a Creative Commons Attribution 4.0 International License, which permits use, sharing, adaptation, distribution and reproduction in any medium or format, as long as you give appropriate credit to the original author(s) and the source, provide a link to the Creative Commons licence, and indicate if changes were made. The images or other third party material in this article are included in the article's Creative Commons licence, unless indicated otherwise in a credit line to the material. If material is not included in the article's Creative Commons licence and your intended use is not permitted by statutory regulation or exceeds the permitted use, you will need to obtain permission directly from the copyright holder. To view a copy of this licence, visit http://creativecommons.org/licenses/by/4.0/. 
communication systems $[3,4]$ and has been discussed in the 6th generation mobile communication system for large-scale access. NOMA adopts superposition coding to realize the transmission of multiple signals in the same time/frequency/code domains; at the receiver, the information required is extracted by the successive interference cancelation (SIC) scheme to improve the utilization of spectrum resources.

The uplink and downlink communications in NOMA system have been studied by many treatises. For downlink communications, the authors in [5] conducted a detailed study on the performance of NOMA with the perfect channel state information (pCSI) condition by analyzing the outage probability. Furthermore, the ergodic rate of NOMA with pCSI was optimized in [6], through which the authors obtained a closed-form globally optimal solution. Considering the low latency in combined with high reliability is a challenging task, the performance of NOMA for URLLC was analyzed in [7], and the results showed that NOMA has reduced the physical layer latency and improved the reliability of supporting the time-critical applications obviously. To draw more conclusions, the authors in [2] proposed a unified framework of NOMA and derived the exact expressions of outage probability as well as the system throughput in delay-limited mode. To study paired users, the authors in [8] developed the optimal and suboptimal schemes. For uplink communications, the performance with pCSI of the NOMA system was characterized by outage probability and achievable sum rate, which is concerned with the power back-off step [9]. To further analyze the performance of uplink NOMA, the authors provide the expressions of effective capacity in [10] and studied the performance of two users under quality of service (QoS) delay constraints. In [11], the authors discussed external and internal eavesdropping scenarios and derived expressions of secrecy outage probability and throughput with pCSI. The impact of pCSI on NOMA systems was investigated carefully in the above researches, while the imperfect channel state information (ipCSI) should be discussed, which is suitable to the practical NOMA scenarios. Based on these, the authors derived a closed-form approximation of the outage probability for users and the high signal-to-noise ratio (SNR) expressions in downlink NOMA with ipCSI in [12]. To prevent the interception of information between base station and receiver, the authors of [13] studied the secrecy outage probability and average secrecy capacity under ipCSI condition. The influence of ipCSI on the security performance of system could be seen when their is the existence of multiple eavesdropping channels.

URLLC is an important application scenario of the 5th generation networks, with new features of high reliability, low latency, and high availability. In [14], the authors proposed a cross-layer optimization framework to ensure that the wireless access network has ultrahigh reliability and ultra-low delay. In order to focus on local communication scenarios, the authors discussed the delay and packet loss of URLLC, as well as the network availability that supports the QoS of users [15]. As cloud capabilities tend toward the network edge, mobile edge computing (MEC) has become a new trend in mitigating the terminal computing abilities. MEC is considered as an effective solution for URLLC, which is capable of reducing the delay of computationally intensive tasks by invoking great computing cells within a short distance [16]. Given that its computing ability is near that of mobile devices and ultra-low latency is its greatest advantage, MEC is widely regarded as the key technology of the next Internet generation [17]. Intelligent applications and networks are deeply integrated based on MEC and Internet of Things to achieve the corresponding requirements of users. The MEC server can be distributed at the edge of networks 
and perform computation-intensive and delay-critical tasks from mobile devices [18, 19]. In view of the ultra-low latency characteristic of MEC, the authors evaluated its actual delay and throughput performance in cellular networks and found that MEC reduces the delay of downlink communication [20]. To improve the efficiency of offloading tasks, the authors of [21] proposed an energy-saving offloading strategy that the computational offloading problem of MEC is transformed into a system cost minimization considering the completion time and energy.

Combining MEC and NOMA is an effective method to utilize computing capacities and improve energy efficiency. The authors in [22] discussed the effect of NOMA on delay and energy efficiency of offloading tasks in MEC, where both uplink and downlink NOMA are taken into consideration. In [23], the authors proposed a NOMA-based computational offloading scheme to reduce the task execution time for users. A distributed algorithm was also proposed to optimize users' transmission time in [24]. The results showed that NOMA-based MEC has more advantages in delay than traditional frequency division multiple access-assisted MEC. In order to reduce the energy consumption of offloading tasks, the authors developed an optimization framework based on NOMA to optimize communication resource allocation and transmission power [25]. In [26], the authors studied energy consumption, where NOMA-based MEC offload scheduling can reduce the system energy consumption compared with OMA. Moreover, the authors in [27] minimized the offloading delay and analyzed the convergence speed.

This treatise focuses on the combination of MEC and NOMA technology under the ipCSI condition in actual scenarios. Based on [28], the NOMA-assisted MEC network is considered where all users transmit the tasks to the MEC server through uplink transmission. The researches in $[29,30]$ illustrate the advantages of combining NOMA with MEC. However, NOMA-MEC is still in its infancy under ipCSI condition. In addition, NOMA has better outage performance than OMA. There are many OMA schemes this paper focuses on comparing NOMA and time division multiple address (TDMA). These are the motivations of this paper. The contributions of this work are summarized as follows:

1) We study the outage performance under two conditions in NOMA-MEC. The NOMA framework studied is applied to the MEC scenario, and the closed-form expressions of the offloading outage probability for paired users (the $m$ th user and the $n$th user) under PCSI and ipCSI conditions through setting the target transmission rate $v_{n}$ and $v_{m}$ are derived. In order to get more conclusions, we also obtain the expressions of the asymptotic offloading outage probability at high SNRs and provide the diversity orders of user. Additionally, we obtain the diversity orders of the both two users are zeros under ipCSI condition. The diversity order of the $n$th user under pCSI is $n$, while that of the $m$ th user is zero.

2) We evaluate theoretical results of system performance by simulation, which shows that the offloading outage probability under ipCSI is larger than that under pCSI. We further analyze the impact of changing channel estimation errors on system performance. With the increasing of channel estimation errors, the offloading outage behaviors for users are becoming more worse. In addition, when the offloading tasks are reduced or the offloading time is increased, the offloading outage probability for the users will be decreased. 
3) We study the throughput and energy efficiency for two users in delay-limited transmission mode of NOMA-MEC system and derive the corresponding expressions. We find that NOMA-MEC has higher system throughput and energy efficiency than TDMA-MEC. In addition, the throughput and energy efficiency under ipCSI are lower than those under pCSI, while the system throughput and energy efficiency will decrease as the channel estimation errors increase.

\section{System model}

Considering NOMA-based MEC communication scenario, $M$ users offload tasks to a single MEC server illustrated in Fig. 1. Assume that each node is a single antenna device and operates in half duplex mode. All communication links in network are subject to Rayleigh fading and disturbed by additive white Gaussian noise (AWGN). $\widehat{h}_{i} \sim \mathcal{C N}\left(0, \widehat{\Omega}_{i}\right)$ denote the channel coefficients of links between the user and the MEC server, where $i \in\{1,2, \cdots, M\} . \widehat{\Omega}_{i}=d_{i}{ }^{-\alpha}$, where $d_{i}$ represents the distance between the user and the server, and $\alpha$ is the path loss exponent. Due to channel estimation errors, it is difficult to obtain the pCSI of channels for NOMA-MEC system in practical communication scenarios. To evaluate the influence of ipCSI in NOMA-MEC system, the channel coefficient is modeled as $\widehat{h}_{i}=h_{i}+\varpi e_{i}$, where $\varpi \in(0,1), h_{i}$ represents the channel gain under the pCSI condition. $\varpi=0$ denotes that the system has ability to obtain the pCSI, and $\varpi=1$ denotes that the system cannot obtain the pCSI and will suffer from the channel estimation error $e_{i} \sim \mathcal{C N}\left(0, \sigma_{e_{i}}^{2}\right)$. Assuming that $h_{i}$ is statistically independent of $e_{i}$, $\gamma_{i}=\sigma_{e_{i}}^{2} / \widehat{\Omega}_{i}$ represents the relative channel estimation error and has $\sigma_{e_{i}}^{2}=\gamma_{i} d_{i}^{-\alpha}$.

In this paper, two users are selected from $M$ users, i.e., the $n$th and $m$ th users for nonorthogonal transmission, where a pair of users simultaneously offload tasks to the MEC server. The channel gains between users and the MEC server are sorted as $\left|\widehat{h}_{m}\right|^{2} \leq\left|\widehat{h}_{n}\right|^{2}$, where the $n$th and $m$ th users have similar channel estimation errors (i.e., $\left|h_{m}\right|^{2} \leq\left|h_{n}\right|^{2}$ ). On the basis of the principle of NOMA, the received expression of offloading tasks at MEC server is given by:

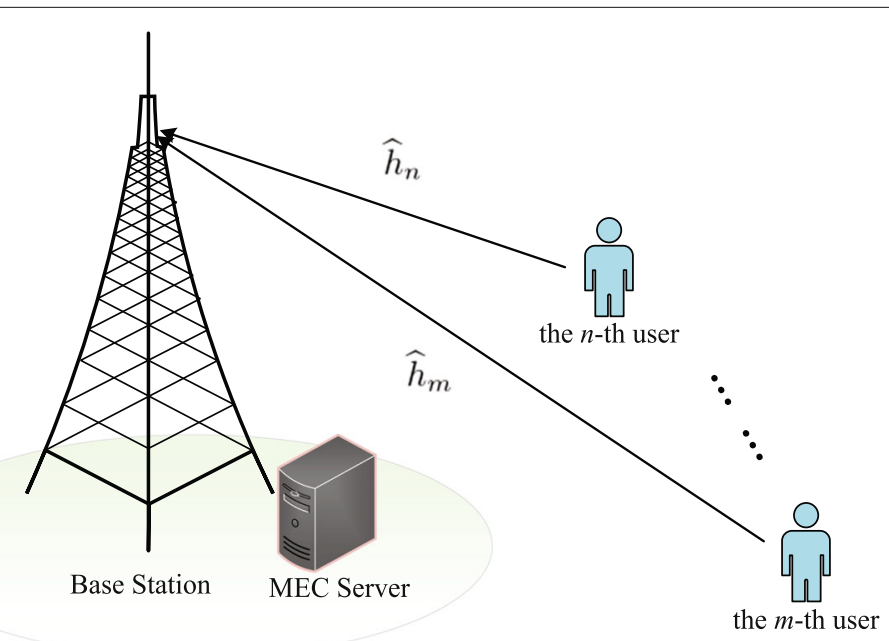

Fig. 1 NOMA-MEC system model 


$$
y_{\mathrm{MEC}}=\left(h_{m}+\varpi e_{m}\right) \sqrt{P_{m}} x_{m}+\left(h_{n}+\varpi e_{n}\right) \sqrt{P_{n}} x_{n}+n_{\mathrm{MEC}},
$$

where $x_{j}$ denotes the offloading task of the $j$ th user, $j \in\{m, n\} . n_{\mathrm{MEC}} \sim \mathcal{C N}\left(0, \sigma_{\mathrm{MEC}}^{2}\right)$ represents the AWGN at the MEC server. The transmission power of the $j$ th user is denoted as $P_{j}$, i.e., $P_{j}=a_{j} P$ and $P$ is the total power of the two users. To guarantee better fairness between the users, assume that $a_{m}>a_{n}$ with $a_{m}+a_{n}=1$. Note that optimal power allocation coefficients [9] can further improve the performance in this system; however, it is beyond the scope of this paper. The $m$ th user has an exclusive time slot in TDMA when offloading the tasks, while the $n$th user will also enter the slot to complete its offloading tasks in NOMA-MEC. The $n$th user does not need additional time slot, which is an advantage of NOMA-MEC compared with TDMA-MEC, thus reducing the offloading delay of the system.

According to the principle of uplink NOMA, the MEC server first decodes the task $x_{m}$ with large power allocation coefficient by treating the task $x_{n}$ with small power allocation coefficient as noise and then subtracts this component. After carrying out SIC procedure, the task $x_{n}$ with small power coefficient can be detected. Hence, the signal-tointerference-plus-noise ratios (SINRs) for the MEC server to decode $x_{m}$ and $x_{n}$ are given by:

$$
\Gamma_{m}=\frac{a_{m} \rho\left|h_{m}\right|^{2}}{a_{n} \rho\left|h_{n}\right|^{2}+\varpi\left(\theta_{n}+\theta_{m}\right)+1},
$$

and

$$
\Gamma_{n}=\frac{a_{n} \rho\left|h_{n}\right|^{2}}{\varpi \theta_{n}+1}
$$

respectively, where $\rho \triangleq \frac{P}{\sigma_{\mathrm{MEC}}^{2}}$ is the transmit SNR, $\theta_{j}=\sigma_{e_{j}}^{2} a_{j} \rho$.

Assuming that the $i$ th user has $N_{i}$-bits tasks and offloads these to the MEC server, where the time required to execute the tasks is $T_{\mathrm{MEC}}=\frac{2 N C}{f_{\mathrm{MEC}}}, N$ represents the total tasks; $C$ is the number of central processing unit (CPU) cycles demanding for computing one input bit, and $f_{\mathrm{MEC}}$ is the CPU frequency at the MEC server.

\section{Performance evaluation}

In this section, the offloading outage performance for the paired users under ipCSI/pCSI conditions in the uplink NOMA-MEC system is analyzed. Firstly, we derive the exact closed-form expressions of the offloading outage probability and the asymptotic offloading outage probability in the high SNR region for the users. Then, so as to further study the outage performance in NOMA-MEC, we obtain the diversity orders and evaluate the performance indicators of users such as system throughput and energy efficiency.

\subsection{Outage probability}

Considering that target rates for two users are determined by their QoS, the offloading outage probability becomes a prime indicator to evaluate the system performance. The offloading outage means that the user cannot complete offloading to the MEC server within the specified time. Hence, in uplink NOMA-MEC scenario, the offloading outage performance for the users under ipCSI/pCSI conditions is analyzed in detail.

When the $n$th user completes $N_{n}$-bits offloading tasks within $T_{1}$, the target transmission rate $v_{n}$ of the $n$th user is denoted by $v_{n}=\frac{N_{n}}{T_{1}}$. Once the actual transmission rate $R_{n}$ is less 
than $v_{n}$, the $n$th user has an outage behavior, and then, the offloading outage probability of the $n$th user under ipCSI is given by:

$$
\begin{aligned}
\mathrm{P}_{\mathrm{ipCSI}}^{n} & =\operatorname{Pr}\left(R_{n}<v_{n}\right) \\
& =\operatorname{Pr}\left[\log \left(1+\Gamma_{n}\right)<v_{n}\right] .
\end{aligned}
$$

Theorem 1 The exact closed-form expression for offloading outage probability of the nth user under ipCSI condition in NOMA-MEC system is given by:

$$
\mathrm{P}_{\mathrm{ipCSI}}^{n}=\frac{M !}{(M-n) !(n-1) !} \sum_{i=0}^{M-n}\left(\begin{array}{c}
M-n \\
i
\end{array}\right) \frac{(-1)^{i}}{n+i}\left(1-e^{-\frac{\kappa\left(\varpi \theta_{n}+1\right)}{a_{n} \rho \Omega_{n}}}\right)^{n+i},
$$

where $\kappa=2^{v_{n}}-1, \varpi=1$.

Proof The SINR of the $n$th user $\Gamma_{n}$ can be obtained by (3), and (4) is rewritten as:

$$
\mathrm{P}_{\mathrm{ipCSI}}^{n}=\operatorname{Pr}\left[\log \left(1+\frac{a_{n} \rho\left|h_{n}\right|^{2}}{\varpi \theta_{n}+1}\right)<v_{n}\right] .
$$

Additionally, the offloading outage probability of the $n$th user is given by:

$$
\mathrm{P}_{\mathrm{ipCSI}}^{n}=\operatorname{Pr}\left(\left|h_{n}\right|^{2}<\frac{\kappa\left(\varpi \theta_{n}+1\right)}{a_{n} \rho}\right) .
$$

$\left|h_{m}\right|^{2}$ and $\left|h_{n}\right|^{2}$ are independent random variables that obey variances $\Omega_{m}$ and $\Omega_{n}$, respectively, and with the aid of order statistics [31] and binomial theorem, the PDF of the $n$th user's sorted channel gain $\left|h_{n}\right|^{2}$ can be expressed as:

$$
f_{\left|h_{n}\right|^{2}}(y)=\frac{M !}{(M-n) !(n-1) !} \frac{1}{\Omega_{n}} \sum_{k=0}^{n-1}\left(\begin{array}{c}
n-1 \\
k
\end{array}\right)(-1)^{k} e^{-\frac{y(M-n+k+1)}{\Omega_{n}}} .
$$

Substituting (8) into (7) and performing some simple operations, we can attain (5), which completes the proof.

Corollary 1 Substituting $\varpi=0$ into (5), the exact closed-form expression for offloading outage probability of the $n$th user under $p$ CSI condition is given by:

$$
\mathrm{P}_{\mathrm{pCSI}}^{n}=\frac{M !}{(M-n) !(n-1) !} \sum_{i=0}^{M-n}\left(\begin{array}{c}
M-n \\
i
\end{array}\right) \frac{(-1)^{i}}{n+i}\left(1-e^{-\frac{\kappa}{a_{n} \rho \Omega_{n}}}\right)^{n+i} .
$$

The offloading outage event of the $m$ th user can be expressed that the MEC server first decodes the task $x_{m}$ by treating the task $x_{n}$. At this moment, an offloading outage event occurs when the actual transmission rate $R_{m}=\log \left(1+\Gamma_{m}\right)$ is lower than the transmission rate $v_{m}\left(v_{m}=\frac{N_{m}}{T_{1}}\right)$. Hence, the offloading outage probability of the $m$ th user with ipCSI can be expressed as:

$$
\mathrm{P}_{\mathrm{ipCSI}}^{m}=\operatorname{Pr}\left(R_{m}<v_{m}\right) .
$$

The offloading outage probability of the $m$ th user in the NOMA-MEC system will be given below. 
Theorem 2 The exact closed-form expression for offloading outage probability of the mth user under ipCSI condition in NOMA-MEC system is given by:

$$
\begin{aligned}
\mathrm{P}_{\mathrm{ipCSI}}^{m}= & \frac{M !}{(M-m) !(m-1) !} \frac{M !}{(M-n) !(n-1) !} \sum_{i=0}^{M-m} \sum_{s=0}^{m+i} \sum_{r=0}^{M-n} \sum_{k=0}^{n+r-1} \\
& \times\left(\begin{array}{c}
M-m \\
i
\end{array}\right)\left(\begin{array}{c}
m+i \\
s
\end{array}\right)\left(\begin{array}{c}
M-n \\
r
\end{array}\right)\left(\begin{array}{c}
n+r-1 \\
k
\end{array}\right) \frac{(-1)^{i+s+r+k}}{m+i} \\
& \times \frac{a_{m} \Omega_{m}}{(1+k) a_{m} \Omega_{m}+s \chi a_{n} \Omega_{n}} e^{-\frac{s \times\left[\omega\left(\theta_{n}+\theta_{m}\right)+1\right]}{a_{m} \rho \Omega_{m}}},
\end{aligned}
$$

where $\chi=2^{v_{m}}-1, \varpi=1$.

Proof See the Appendix.

Corollary 2 By substituting $\varpi=0$ into (11), the exact closed-form expression for offloading outage probability of the mth user under pCSI condition is given by:

$$
\begin{aligned}
\mathrm{P}_{\mathrm{pCSI}}^{m}= & \frac{M !}{(M-m) !(m-1) !} \frac{M !}{(M-n) !(n-1) !} \sum_{i=0}^{M-m} \sum_{s=0}^{m+i} \sum_{r=0}^{M-n} \sum_{k=0}^{n+r-1} \\
& \times\left(\begin{array}{c}
M-m \\
i
\end{array}\right)\left(\begin{array}{c}
m+i \\
s
\end{array}\right)\left(\begin{array}{c}
M-n \\
r
\end{array}\right)\left(\begin{array}{c}
n+r-1 \\
k
\end{array}\right) \frac{(-1)^{i+s+r+k}}{m+i} \\
& \times \frac{a_{m} \Omega_{m}}{(1+k) a_{m} \Omega_{m}+s \chi a_{n} \Omega_{n}} e^{-\frac{s \chi}{a_{m} \rho \Omega_{m}}} .
\end{aligned}
$$

\subsection{Diversity order}

In this subsection, we obtain the diversity orders of users under the different channel state conditions, which is defined as follows:

$$
\mu=-\lim _{\rho \rightarrow \infty} \frac{\log \left[\mathrm{P}^{\infty}(\rho)\right]}{\log \rho},
$$

where $\mathrm{P}^{\infty}(\rho)$ represents the offloading outage probability at high SNR of the users.

Corollary 3 When $\rho \rightarrow \infty$ is substituted into (5), with $\rho \rightarrow \infty(x \rightarrow 0), 1-e^{-x} \sim x$, and the nth user's asymptotic offloading outage probability in the high SNR region under the ipCSI condition is given by:

$$
\mathrm{P}_{\mathrm{ipCSI}}^{n, \infty}=\frac{M !}{(M-n) ! n !}\left(\frac{\kappa\left(\theta_{n}+1\right)}{a_{n} \rho \Omega_{n}}\right)^{n} .
$$

Remark 1 Substituting (14) into (13), the diversity order for the nth user under ipCSI condition $\mu_{\mathrm{ipCSI}}^{n}=0$ can be obtained.

Corollary 4 Substituting $\rho \rightarrow \infty$ into (9), the nth user's asymptotic offloading outage probability in the high SNR region under the pCSI condition is given by:

$$
\mathrm{P}_{\mathrm{pCSI}}^{n, \infty}=\frac{M !}{(M-n) ! n !}\left(\frac{\kappa}{a_{n} \rho \Omega_{n}}\right)^{n} .
$$

Remark 2 Substituting (15) into (13), the diversity order for the nth user under $p C S I$ condition $\mu_{\mathrm{pCSI}}^{n}=n$ can be obtained. 
Corollary 5 Substituting $\rho \rightarrow \infty$ into (11), the mth user's asymptotic offloading outage probability in the high SNR region under the ipCSI condition is given by:

$$
\begin{aligned}
\mathrm{P}_{\mathrm{ipCSI}}^{m, \infty}= & \frac{M !}{(M-m) !(m-1) !} \frac{M !}{(M-n) !(n-1) !} \sum_{i=0}^{M-m} \sum_{s=0}^{m+i} \sum_{r=0}^{M-n} \sum_{k=0}^{n+r-1} \\
& \times\left(\begin{array}{c}
M-m \\
i
\end{array}\right)\left(\begin{array}{c}
m+i \\
s
\end{array}\right)\left(\begin{array}{c}
M-n \\
r
\end{array}\right)\left(\begin{array}{c}
n+r-1 \\
k
\end{array}\right) \frac{(-1)^{i+s+r+k}}{m+i} \\
& \times \frac{a_{m} \Omega_{m}}{(1+k) a_{m} \Omega_{m}+s \chi a_{n} \Omega_{n}}\left(1-\frac{s \chi\left(\theta_{n}+\theta_{m}+1\right)}{a_{m} \rho \Omega_{m}}\right) .
\end{aligned}
$$

Proof When $\rho \rightarrow \infty(x \rightarrow 0)$, we have $e^{-\frac{s x\left(\theta_{n}+\theta_{m}+1\right)}{a_{m} \rho \Omega_{m}}} \sim 1-\frac{s \chi\left(\theta_{n}+\theta_{m}+1\right)}{a_{m} \rho \Omega_{m}}$. By substituting it into (11), (16) can be determined. The proof is completed.

Remark 3 Substituting (16) into (13), the diversity order for the mth user under ipCSI condition $\mu_{\mathrm{ipCSI}}^{m}=0$ can be obtained.

Corollary 6 Substituting $\rho \rightarrow \infty$ into (12), the mth user's asymptotic offloading outage probability in the high SNR region under the $p C S I$ condition is given by:

$$
\begin{aligned}
\mathrm{P}_{\mathrm{pCSI}}^{m, \infty}= & \frac{M !}{(M-m) !(m-1) !} \frac{M !}{(M-n) !(n-1) !} \sum_{i=0}^{M-m} \sum_{s=0}^{m+i} \sum_{r=0}^{M-n} \sum_{k=0}^{n+r-1} \\
& \times\left(\begin{array}{c}
M-m \\
i
\end{array}\right)\left(\begin{array}{c}
m+i \\
s
\end{array}\right)\left(\begin{array}{c}
M-n \\
r
\end{array}\right)\left(\begin{array}{c}
n+r-1 \\
k
\end{array}\right) \frac{(-1)^{i+s+r+k}}{m+i} \\
& \times \frac{a_{m} \Omega_{m}}{(1+k) a_{m} \Omega_{m}+s \chi a_{n} \Omega_{n}}\left(1-\frac{s \chi}{a_{m} \rho \Omega_{m}}\right) .
\end{aligned}
$$

Remark 4 Substituting (17) into (13), the diversity order for the mth user under $p C S I$ condition $\mu_{\mathrm{pCSI}}^{m}=0$ can be obtained.

\subsection{Throughput analysis}

In this subsection, the system throughput of NOMA-MEC in the delay-limited transmission mode is discussed. The paired users offload tasks to the MEC server at constant rates of $v_{m}$ and $v_{n}$, respectively.

Under the condition of channel estimation error, the throughput for the users in NOMA-MEC system can be expressed as:

$$
R_{\mathrm{ipCSI}}=\left(1-\mathrm{P}_{\mathrm{ipCSI}}^{n}\right) v_{n}+\left(1-\mathrm{P}_{\mathrm{ipCSI}}^{m}\right) v_{m},
$$

where $\mathrm{P}_{\mathrm{ipCSI}}^{n}$ and $\mathrm{P}_{\mathrm{ipCSI}}^{m}$ have been derived in (5) and (11), respectively.

In the absence of channel estimation error, the throughput for the users in NOMA-MEC system can be expressed as:

$$
R_{\mathrm{pCSI}}=\left(1-\mathrm{P}_{\mathrm{pCSI}}^{n}\right) v_{n}+\left(1-\mathrm{P}_{\mathrm{pCSI}}^{m}\right) v_{m},
$$

where $\mathrm{P}_{\mathrm{pCSI}}^{n}$ and $\mathrm{P}_{\mathrm{pCSI}}^{m}$ have been derived in (9) and (12), respectively. 


\subsection{Energy efficiency}

In this subsection, the energy efficiency in NOMA-MEC system is analyzed based on the system throughput analysis above. Energy efficiency [32] is defined as:

$$
\eta=\frac{\text { Total data rate }}{\text { Total energy consumption }} \text {. }
$$

In this system, the total data rate is expressed as the corresponding system throughput, and the total energy consumption can be expressed as the sum of two users' transmitted power. According to the results derived above, the system energy efficiency under the ipCSI and pCSI conditions is expressed as follows:

$$
\eta_{\text {ipCSI }}=\frac{R_{\text {ipCSI }}}{T P}
$$

and

$$
\eta_{\mathrm{pCSI}}=\frac{R_{\mathrm{pCSI}}}{T P}
$$

respectively, where $T$ represents the transmission time of the entire offloading process and $\eta_{\mathrm{ipCSI}}$ and $\eta_{\mathrm{pCSI}}$ are the energy efficiency of the system with or without channel estimation errors respectively in the delay-limited transmission mode.

\section{Results and discussion}

In this section, the numerical results are given to verify the above theoretical expressions derived. The performance under the ipCSI and pCSI conditions in NOMA-MEC system is further evaluated. Assume that the distance from the MEC server to the $n$th user is $d_{n}=0.3 \mathrm{~m}$, while the distance from the $m$ th user is $d_{m}=0.7 \mathrm{~m}$. The path loss exponent is set to $\alpha=2$, and the power allocation factors are $a_{n}=0.2$ and $a_{m}=0.8$. We assume that the target transmission rates of this system are set to $v_{n}=3 \mathrm{bit} / \mathrm{s}$ and $v_{m}=0.1 \mathrm{bit} / \mathrm{s}$, respectively. Compared with the performance of the traditional OMA, the entire communication process of TDMA is completed in two time slots. In other words, the $m$ th and $n$th users occupy one time slot each in the system.

Figure 2 depicts the offloading outage probability for the two users versus the transmit SNR while the channel estimation errors are $\sigma_{e_{n}}^{2}=-10 \mathrm{~dB}$ and $\sigma_{e_{m}}^{2}=0 \mathrm{~dB}$. The exact theoretical curves for the offloading outage probability of the two users under the ipCSI/pCSI conditions are plotted according to (5), (9) and (11), (12), respectively. It is clear that the exact curves clearly match the simulation curves. The offloading outage probability of the $m$ th user is lower than the $n$th user's probability at low SNR, and the opposite is true at the high SNR. Error floors exit with the users under the ipCSI condition because of the interference of channel estimation errors during transmission. Meanwhile, the offloading performance of the $n$th user is higher than that in TDMA-MEC under the same conditions. Hence, the existence of channel estimation errors must be considered in the actual NOMA-MEC scenarios.

As shown in Fig. 3, we present the system throughput versus the SNR under ipCSI/pCSI conditions in delay-limited transmission mode, and the channel estimation errors are $\sigma_{e_{n}}^{2}=0 \mathrm{~dB}$ and $\sigma_{e_{m}}^{2}=-10 \mathrm{~dB}$. The solid curves are the throughput in the NOMA-MEC system with or without channel estimation error, in which obtained according to (18) and (19). The dashed curve represents the throughput in the TDMA-MEC system with or without channel estimation error. It is observed that with increasing the $\sigma_{e_{i}}^{2}$, the system throughput of TDMA-MEC with ipCSI is becoming much smaller. This is due to the fact 


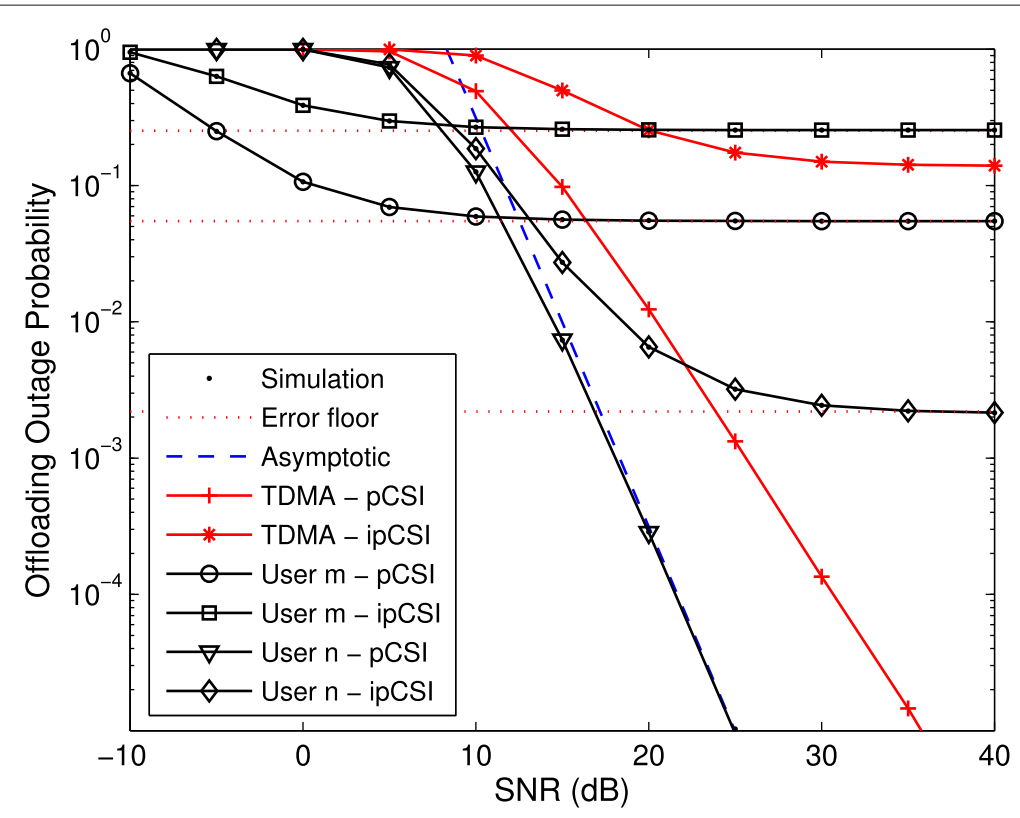

Fig. 2 Offloading outage probability for two users versus SNR

that the channel estimate error $\sigma_{e_{i}}^{2}$ leads to the worse offloading outage probability. Additionally, we can observe that channel estimation errors affect the performance index of this system, because the offloading outage probability for the users under pCSI is lower than that under ipCSI. The results show that with the $\sigma_{e_{n}}^{2}$ value increases, the offloading outage probability of the users increases, but the system throughput at the high SNR region decreases.

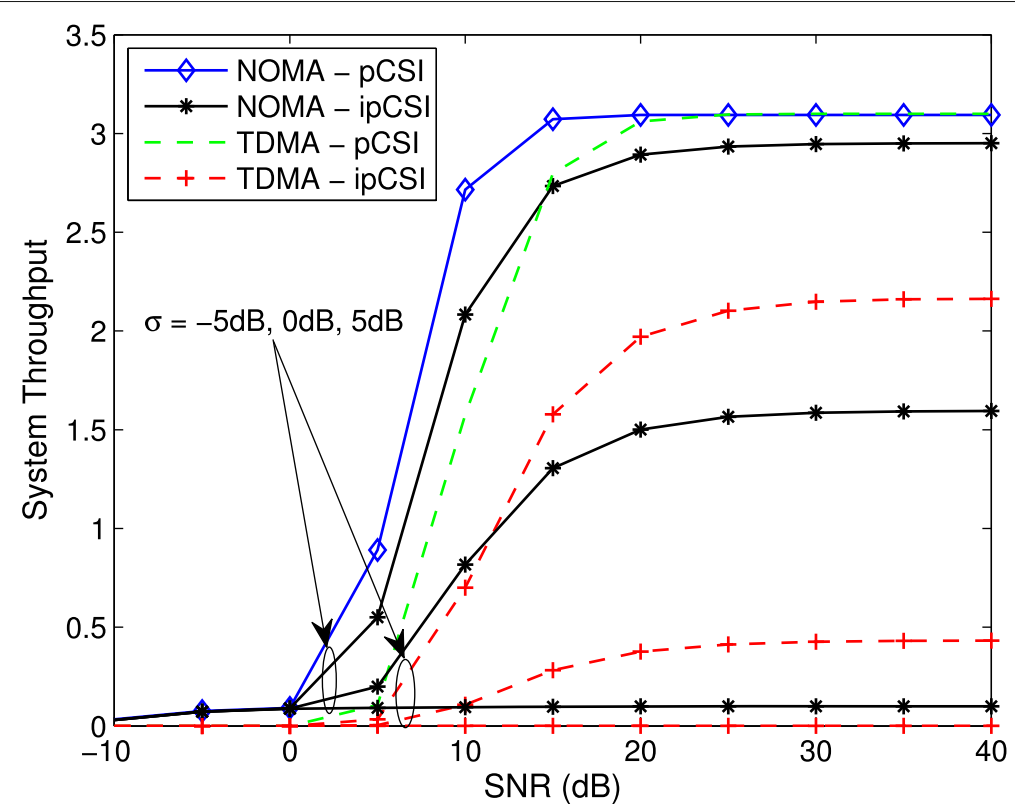

Fig. 3 System throughput versus SNR under ipCSI/pCSI conditions in delay-limited transmission mode 


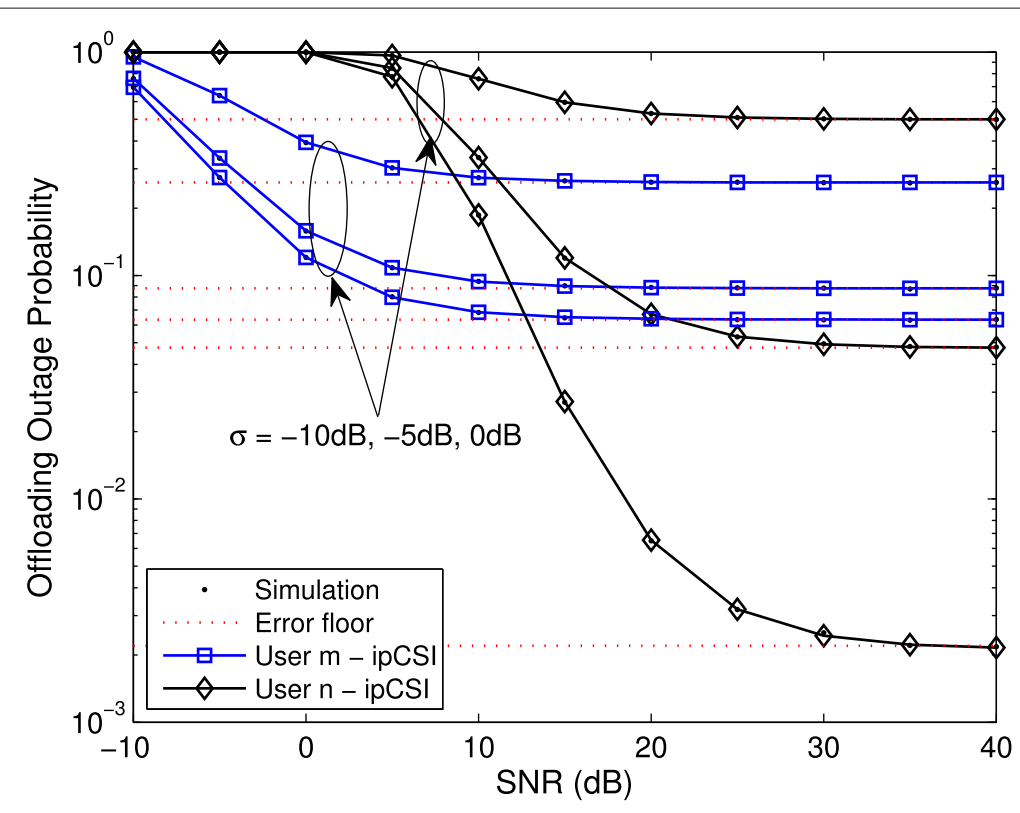

Fig. 4 Offloading outage probability versus SNR with various values of channel estimation errors

In Fig. 4, the offloading outage probability for the two users with channel estimation errors from $\sigma_{e_{n}}^{2}=\sigma_{e_{m}}^{2}=0 \mathrm{~dB}$ to $\sigma_{e_{n}}^{2}=\sigma_{e_{m}}^{2}=-10 \mathrm{~dB}$ is shown. We can observe that error floors exist under the ipCSI condition, which verify the conclusions in Remark 1 and Remark 3 . The offloading outage probability gradually increases with the increase of the channel estimation error values. We can also see that the impact on the $n$th user is more obvious than the $m$ th user because of the interference of the $n$th user and the channel

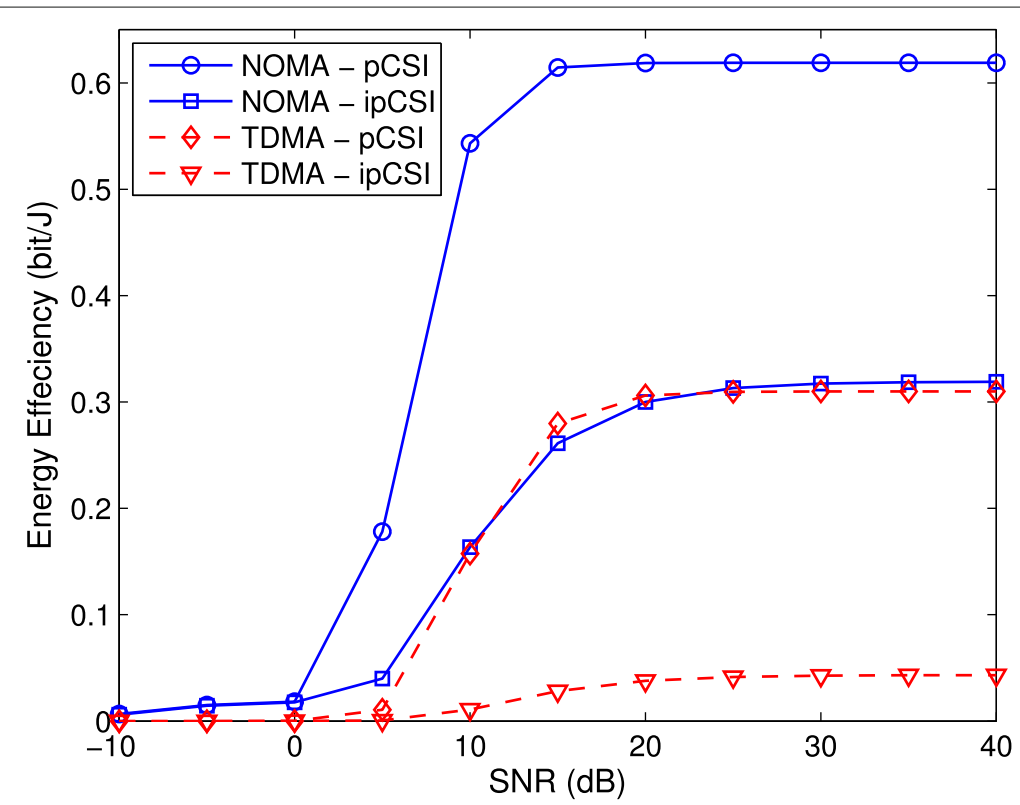

Fig. 5 System energy efficiency versus SNR under ipCSI/pCSI conditions in delay-limited transmission mode, $P=5 \mathrm{~W}$, and $T=1 \mathrm{~S}$ 


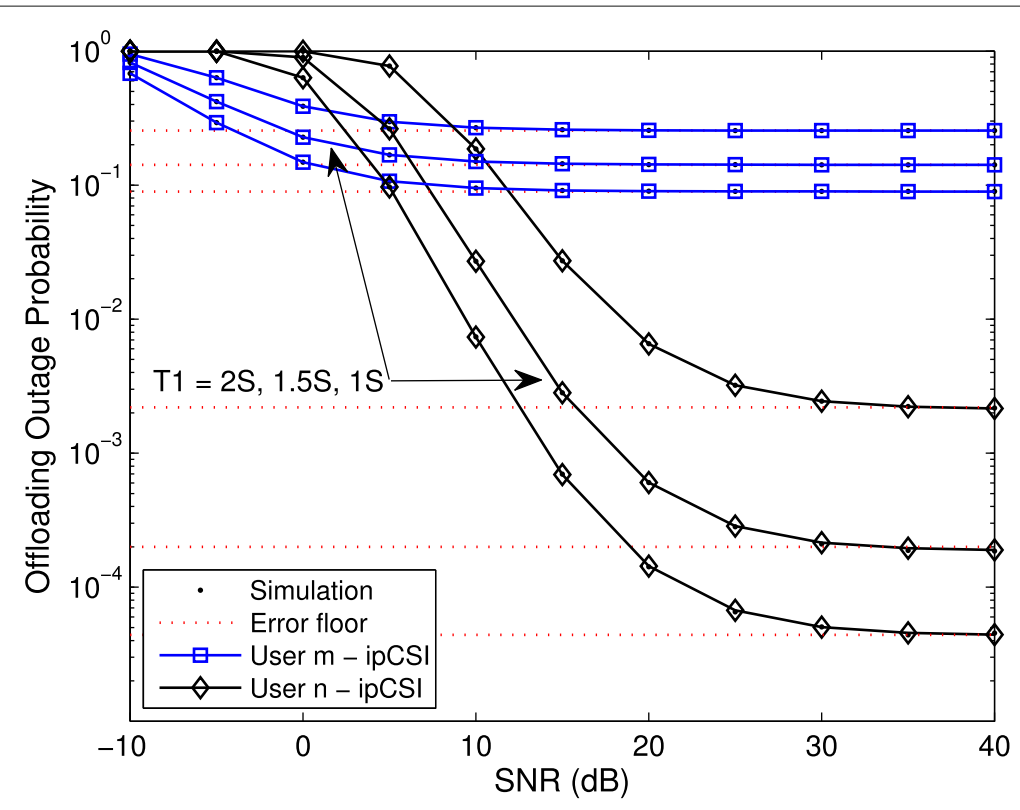

Fig. 6 Offloading outage probability versus SNR with various values of offloading time

estimation error. By contrast, the $n$th user is only interfered by the channel estimation error.

In Fig. 5, the system energy efficiency versus the SNR for the two users under ipCSI/pCSI conditions in delay-limited transmission mode is shown. The solid curves represent the energy efficiency for the NOMA-MEC system which are obtained from (18), (21) and (19), (22) with the throughput. The energy efficiency for the NOMA-MEC system is much higher than that of TDMA-MEC. At high SNR, the energy efficiency of

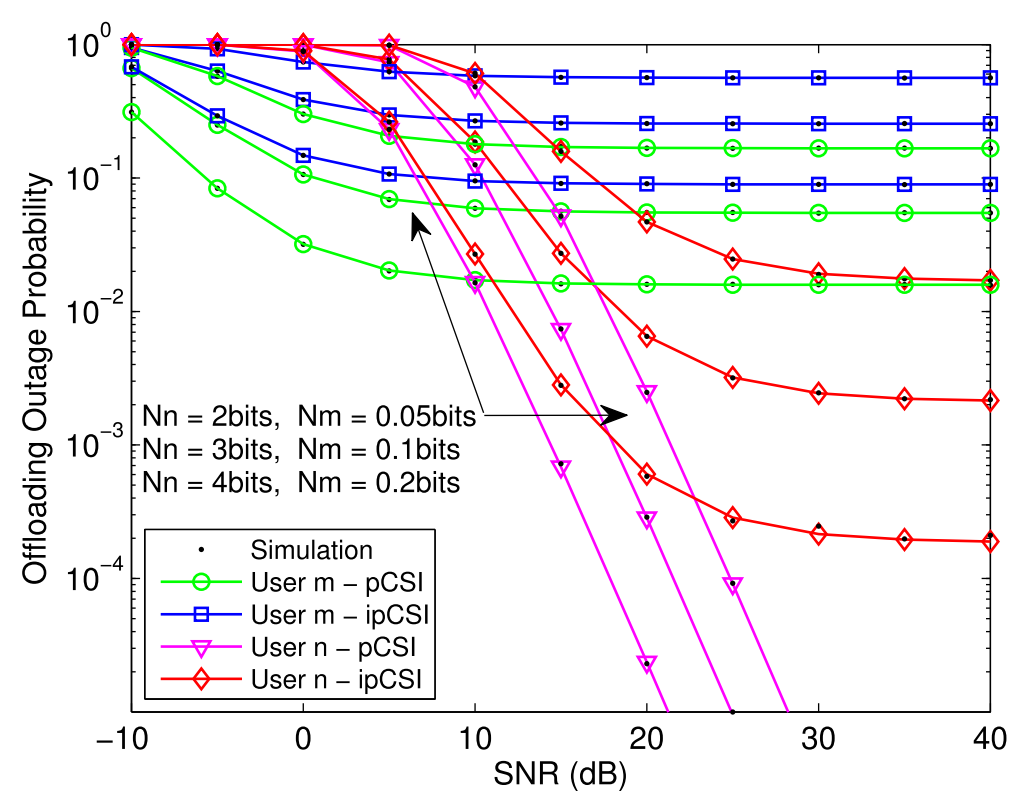

Fig. 7 Offloading outage probability versus SNR with various values of tasks 


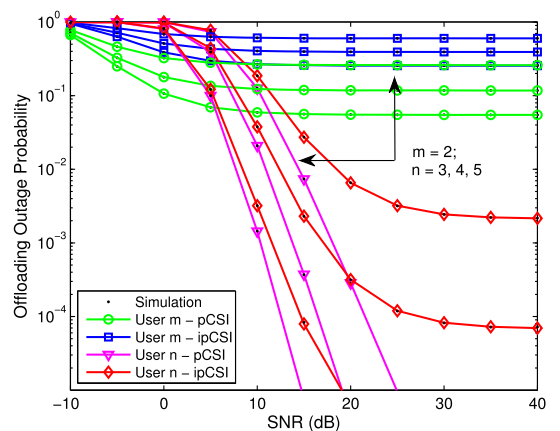

(a)

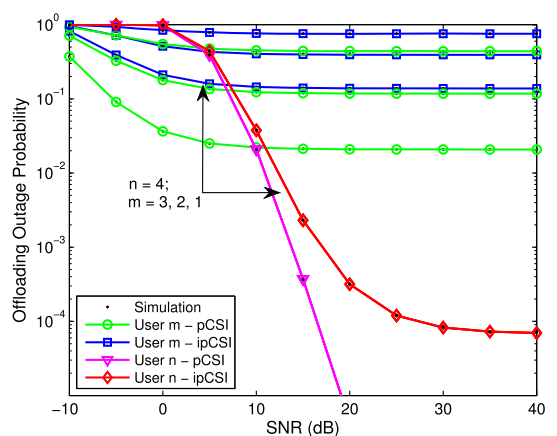

(b)

Fig. 8 Offloading outage probability versus SNR with various values of the ith user

the NOMA-MEC system with channel estimation error is higher than that of the TDMAMEC system without channel estimation error, because NOMA-ipCSI can achieve greater throughput than TDMA-pCSI in such transmission mode.

In Fig. 6, the offloading outage probability for the two users versus offloading times from $1 \mathrm{~S}$ to $2 \mathrm{~S}$ is shown. It is clear that when users are allowed less time to offload, the offloading outage probabilities will be increased. This is due to the smaller the offloading time is, the higher the target transmission rate of the users, and the greater the offloading outage probability is. Therefore, the offloading time must be considered in actual NOMAMEC systems.

In Fig. 7, we present the offloading outage probability for the two users versus various values of tasks. At $N_{n}=4$ bits, $N_{m}=0.2$ bits; $N_{n}=3$ bits, $N_{m}=0.1$ bits; and $N_{n}=2$ bits, $N_{m}=0.05$ bits, we can observe that with offloading tasks of both the $n$th and $m$ th users increase simultaneously, the offloading outage probability also increases gradually. This is because that with the amount of tasks increases, the requirements for system performance are becoming higher. Hence, it is also necessary to consider the offloading tasks in NOMA-MEC.

Figure 8 plots the offloading outage probabilities for the two users versus various values of the $i$ th user. In Fig. $8 \mathrm{a}, m=2$ and the values of $n$ are 3, 4, and 5, while in Fig. $8 \mathrm{~b}, n=4$ and the values of $m$ are 1,2 , and 3 . We can observe that when the user is closer to the MEC server, the outage probability is becoming smaller. This is consistent with the fact that MEC is closer to the mobile devices.

\section{Conclusions}

We have investigated the offloading performance of uplink NOMA-based MEC with ipCSI/pCSI. The exact and asymptotic expressions of offloading outage probability for the paired users were derived in detail. The analytical results have shown that the offloading probability of NOMA-MEC with pCSI is superior to TDMA-MEC. As a result of channel estimation errors, the offloading behaviors of NOMA-MEC with ipCSI are worse than that of pCSI. When the channel estimation errors increase, the offloading outage probability of NOMA-MEC is becoming larger. Finally, the throughput and energy efficiency of NOMA-MEC have been investigated with ipCSI/pCSI. In addition, the impact 
on the outage behaviors for users when the offloading time or tasks change has also been discussed.

\section{Appendix: Proof of Theorem 2}

By substituting (2) into (10), the offloading outage probability $\mathrm{P}_{\mathrm{ipCSI}}^{m}$ can be given by:

$$
\mathrm{P}_{\mathrm{iPCSI}}^{m}=\operatorname{Pr}\left[\log \left(1+\frac{a_{m} \rho\left|h_{m}\right|^{2}}{a_{n} \rho\left|h_{n}\right|^{2}+\varpi\left(\theta_{n}+\theta_{m}\right)+1}\right)<v_{m}\right]
$$

Furthermore, the above equation can be calculated as:

$$
\begin{aligned}
\mathrm{P}_{\mathrm{ipCSI}}^{m} & =\operatorname{Pr}\left(\left|h_{m}\right|^{2}<\frac{\chi\left[a_{n} \rho\left|h_{n}\right|^{2}+\varpi\left(\theta_{n}+\theta_{m}\right)+1\right]}{a_{m} \rho}\right) \\
& =\int_{0}^{\infty} f_{\left|h_{n}\right|^{2}}(y) d y \int_{0}^{\frac{\chi\left[a_{n} \rho\left|h_{n}\right|^{2}+\varpi\left(\theta_{n}+\theta_{m}\right)+1\right]}{a_{m} \rho}} f_{\left|h_{m}\right|^{2}}(x) d x .
\end{aligned}
$$

With some arithmetic operations, the above expression can be given by:

$$
\mathrm{P}_{\mathrm{ipCSI}}^{m}=\int_{0}^{\infty} f_{\left|h_{n}\right|^{2}}(y)\left[F_{\left|h_{m}\right|^{2}}\left(\frac{\chi\left[a_{n} \rho y+\varpi\left(\theta_{n}+\theta_{m}\right)+1\right]}{a_{m} \rho}\right)\right] d y .
$$

$\left|h_{m}\right|^{2}$ and $\left|h_{n}\right|^{2}$ are independent random variables that obey variances $\Omega_{m}$ and $\Omega_{n}$, respectively, and with the aid of order statistics and binomial theorem, the CDF of the $m$ th user's sorted channel gain $\left|h_{m}\right|^{2}$ can be expressed as:

$$
F_{\left|h_{m}\right|^{2}}(x)=\frac{M !}{(M-m) !(m-1) !} \sum_{i=0}^{M-m}\left(\begin{array}{c}
M-m \\
i
\end{array}\right) \frac{(-1)^{i}}{m+i}\left(1-e^{-\frac{x}{\Omega m}}\right)^{m+i} .
$$

Substituting (26) into (25), the offloading outage probability of the $m$ th user is given by:

$$
\begin{aligned}
\mathrm{P}_{\mathrm{ipCSI}}^{m}= & \frac{M !}{(M-m) !(m-1) !} \sum_{i=0}^{M-m} \sum_{s=0}^{m+i}\left(\begin{array}{c}
M-m \\
i
\end{array}\right)\left(\begin{array}{c}
m+i \\
s
\end{array}\right) \\
& \times \frac{(-1)^{i+s}}{m+i} \int_{0}^{\infty} f_{\left|h_{n}\right|^{2}}(y) e^{-\frac{s \chi\left[a_{n} \rho y+\varpi\left(\theta_{n}+\theta_{m}\right)+1\right]}{a_{m} \rho \Omega_{m}}} d y .
\end{aligned}
$$

The PDF of the $n$th user's sorted channel gain $\left|h_{n}\right|^{2}$ is known, and substituting it into the above expression can obtain the offloading outage probability as follows:

$$
\begin{aligned}
\mathrm{P}_{\mathrm{ipCSI}}^{m}= & \frac{M !}{(M-m) !(m-1) !} \frac{M !}{(M-n) !(n-1) ! \Omega_{n}} \sum_{i=0}^{M-m} \sum_{s=0}^{m+i} \sum_{r=0}^{M-n} \\
& \times \sum_{k=0}^{n+r-1}\left(\begin{array}{c}
M-m \\
i
\end{array}\right)\left(\begin{array}{c}
m+i \\
s
\end{array}\right)\left(\begin{array}{c}
M-n \\
r
\end{array}\right)\left(\begin{array}{c}
n+r-1 \\
k
\end{array}\right) \\
& \times \frac{(-1)^{i+s+r+k}}{m+i} \int_{0}^{\infty} \mathrm{e}^{-\frac{(1+k) y}{\Omega_{n}}} e^{-\frac{s \times\left[a_{n} \rho y+\varpi\left(\theta_{n}+\theta_{m}\right)+1\right]}{a_{m} \rho \Omega_{m}}} d y .
\end{aligned}
$$

By sorting the above expression further, (11) can be attained easily. The proof is completed.

\section{Abbreviations}

URLLC: Ultra-reliable and low latency communications; OMA: Orthogonal multiple access; NOMA: Non-orthogonal multiple access; SIC: Successive interference cancelation; pCSI: Channel state information; QoS: Quality of service; ipCSI: Imperfect channel state information; MEC: Mobile edge computing; SNR: Signal-to-noise ratio; AWGN: Additive white Gaussian noise; SINR: Signal-to-interference-plus-noise ratio; CPU: Central processing unit; TDMA: Time division multiple address 


\section{Acknowledgements}

The authors thank the anonymous reviewers for their constructive comments and suggestions. This work is supported in part by the Natural Science Foundation of Beijing Municipality under grants 4204099, 19L2022, L182032, L182039, and KZ201911232046; in part by the Science and Technology Project of Beijing Municipal Education Commission under grants KM202011232002 and KM202011232003; in part by the Key Research and Cultivation Project at Beijing Information Science and Technology University under grants 5211910924 and 5211910926; and in part by the Supplementary and Supportive Project for Teachers at Beijing Information Science and Technology University under grants 5111911147 and 5029011103.

\section{Authors' contributions}

All authors contributed to the design and analysis of the research, to the simulation results, and to the writing of the manuscript. All authors read and approved the final manuscript.

\section{Competing interests}

The authors declare that they have no competing interests.

Received: 26 February 2020 Accepted: 10 June 2020

Published online: 01 July 2020

\section{References}

1. C. Y. She, R. Dong, Z. Y. Gu, Z. W. Hou, Y. H. Li, W. Hardjawana, C. Y. Yang, L. Y. Song, V. B, Deep learning for ultra-reliable and low-latency communications in 6G networks. IEEE, Networks,. https://arxiv.org/abs/2002.11045. Accessed 22 Feb 2020

2. X. W. Yue, Z. J. Qin, Y. W. Liu, S. L. Kang, Y. Chen, A unified framework for non-orthogonal multiple access. IEEE Trans. Commun. 66(11), 5346-5359 (2018)

3. Z. G. Ding, Y. W. Liu, J. Choi, Q. Sun, M. Elkashlan, I. Chin-Lin, H. V. Poor, Application of non-orthogonal multiple access in LTE and 5G networks. IEEE Commun. Mag. 55(2), 185-191 (2017)

4. Y. W. Liu, Z. J. Qin, M. Elkashlan, Z. G. Ding, A. Nallanathan, L. Hanzo, Non-orthogonal multiple access for 5G and beyond. arXiv preprint arXiv:1808.00277 (2018)

5. Z. G. Ding, Z. Yang, P. Z. Fan, H. V. Poor, On the performance of non-orthogonal multiple access in 5 G systems with randomly deployed users. IEEE Sig. Process Lett. 21(12), 1501-1505 (2014)

6. L. P. Zhu, J. Zhang, Z. Y. Xiao, X. B. Cao, D. O. Wu, Optimal user pairing for downlink non-orthogonal multiple access (NOMA). IEEE Wirel. Commun. Lett. 8(2), 328-331 (2018)

7. M. Amjad, L. Musavian, in IEEE Globecom Workshops (GC Wkshps), Performance analysis of NOMA for ultra-reliable and low-latency communications (IEEE, Abu Dhabi, 2018), pp. 1-5

8. J. M. Kang, I. M. Kim, Optimal user grouping for downlink NOMA. IEEE Wirel. Commun. Lett. 7(5), 724-727 (2018)

9. N. B. Zhang, J. Wang, G. X. Kang, Y. Liu, Uplink nonorthogonal multiple access in 5 G systems. IEEE Commun. Lett. 20(3), 458-461 (2016)

10. R. Nasfi, A. Chorti, in Eleventh International Conference on Ubiquitous and Future Networks (ICUFN), Performance analysis of the uplink of a two user NOMA network under QoS delay constraints (IEEE, Zagreb, 2019), pp. 526-528

11. X. W. Yue, Y. W. Liu, Y. Y. Yao, X. H. Li, R. Liu, A. Nallanathan, Secure communications in a unified non-orthogonal multiple access framework. IEEE Trans. Wirel. Commun. 19(3), 2163-2178 (2020)

12. Z. Yang, Z. G. Ding, P. Z. Fan, G. K. Karagiannidis, On the performance of non-orthogonal multiple access systems with partial channel information. IEEE Trans. Commun. 64(2), 654-667 (2016)

13. G. F. Pan, H. J. Lei, Y. S. Deng, L. S. Fan, J. Yang, Y. F. Chen, Z. G. Ding, On secrecy performance of MISO SWIPT systems with TAS and imperfect CSI. IEEE Trans. Commun. 64(9), 3831-3843 (2016)

14. C. Y. She, C. Y. Yang, T. Q. S. Quek, Cross-layer optimization for ultrareliable and low-latency radio access networks. IEEE Trans. Wirel. Commun. 17(1), 127-141 (2018)

15. C. Y. She, C. Y. Yang, T. Q. S. Quek, Radio resource management for ultra-reliable and low-latency communications. IEEE Commun. Mag. 55(6), 72-78 (2017)

16. J. H. Liu, Q. Zhang, Offloading schemes in mobile edge computing for ultra-reliable low latency communications. IEEE Access. 6, 12825-12837 (2018)

17. S. Wang, X. Zhang, Y. Zhang, L. Wang, J. W. Yang, W. B. Wang, A survey on mobile edge networks: convergence of computing, caching and communications. IEEE Access. 5, 6757-6779 (2017)

18. M. Chiang, T. Zhang, Fog and IoT: an overview of research opportunities. IEEE Internet Things J. 3(6), 854-864 (2016)

19. G. P. Fettweis, The tactile internet: applications and challenges. IEEE Veh. Technol. Mag. 9(1), 64-70 (2014)

20. K. Amemiya, Y. Akiyama, K. Kobayashi, Y. Inoue, S. Yamamoto, A. Nakao, in IEEE 7th International Conference on Cloud Networking (CloudNet), On-site evaluation of a software cellular based MEC system with downlink slicing technology (IEEE, Tokyo, 2018), pp. 1-7

21. H. Y. Yu, Q. Y. Wang, S. T. Guo, in IEEE International Conference on Networking, Architecture and Storage (NAS), Energy-efficient task offloading and resource scheduling for mobile edge computing (IEEE, Chongqing, 2018), pp. 1-4

22. Z. G. Ding, P. Z. Fan, H. V. Poor, Impact of non-orthogonal multiple access on the offloading of mobile edge computing. IEEE Trans. Commun. 67(1), 375-390 (2018)

23. F. Wang, X. Zhang, in IEEE International Conference on Communications (ICC), Dynamic computation offloading and resource allocation over mobile edge computing networks with energy harvesting capability (IEEE, Kansas, 2018), pp. 1-6

24. Y. Wu, K. J. Ni, C. Zhang, L. P. Qian, D. H. K. Tsang, Noma-assisted multi-access mobile edge computing: a joint optimization of computation offloading and time allocation. IEEE Trans. Veh. Technol. 67(12), 12244-12258 (2018)

25. A. Kiani, N. Ansari, Edge computing aware NOMA for 5 G networks. IEEE Internet Things J. 5(2), 1299-1306 (2018) 
26. Y. J. Pan, M. Chen, Z. H. Yang, N. Huang, M. Shikh-Bahaei, Energy-efficient NOMA-based mobile edge computing offloading. IEEE Commun. Lett. 23(2), 310-313 (2018)

27. Z. G. Ding, D. W. K. Ng, R. Schober, H. V. Poor, Delay minimization for NOMA-MEC offloading. IEEE Sig. Process. Lett. 25(12), 1875-1879 (2018)

28. Y. H. Ye, G. Y. Lu, R. Q. Hu, L. Q. Shi, in IEEE International Conference on Communications Workshops (ICC Workshops), On the performance and optimization for MEC networks using uplink NOMA (IEEE, Shanghai, 2019), pp. 1-6

29. P. T. Yang, L. X. Li, W. Liang, H. S. Zhang, Z. G. Ding, in 28th Wireless and Optical Communications Conference (WOCC), Latency optimization for multi-user NOMA-MEC offloading using reinforcement learning (IEEE, Beijing, 2019), pp. 1-5

30. X. B. Diao, J. C. Zheng, Y. Wu, Y. M. Cai, Joint computing resource, power, and channel allocations for D2D-assisted and NOMA-based mobile edge computing. IEEE Access. 7, 9243-9257 (2019)

31. H. A. David, H. N. Nagaraja, Order Statistics. (John Wiley, New York, 2004)

32. X. W. Yue, Y. W. Liu, S. L. Kang, A. Nallanathan, Z. G. Ding, Exploiting full/half-duplex user relaying in NOMA systems. IEEE Trans. Commun. 66(2), 560-575 (2018)

\section{Publisher's Note}

Springer Nature remains neutral with regard to jurisdictional claims in published maps and institutional affiliations.

\section{Submit your manuscript to a SpringerOpen ${ }^{\odot}$ journal and benefit from:}

- Convenient online submission

Rigorous peer review

Open access: articles freely available online

- High visibility within the field

- Retaining the copyright to your article

Submit your next manuscript at $\gg$ springeropen.com 